\title{
FREE QUASICONFORMALITY IN BANACH SPACES I
}

\section{Jussi Väisälä}

\section{Introduction}

1.1. The idea of the paper. The concept of a quasiconformal map was introduced in 1928 by H. Grötzsch [Gr], who considered maps in the plane $R^{2}$. The notion was later extended to maps in $R^{n}$ by several authors. The purpose of this article is to lay foundations of an analogous theory in general Banach spaces. The main emphasis will be on the basic definitions and on relationships with various known classes such as the quasisymmetric maps. The methods of this paper are elementary. In a later paper [V $\ddot{a}_{4}$ ] we shall consider the boundary behavior and other more specific properties of these maps. Some of these results are announced in Section 7.

Let $E$ and $E^{\prime}$ be real Banach spaces, and let $G \subset E$ and $G^{\prime} \subset E^{\prime}$ be domains, that is, open connected nonempty sets. Let $\varphi:[0, \infty) \rightarrow[0, \infty)$ be a homeomorphism with $\varphi(t) \geq t$. In Section 3 we shall define the class of freely $\varphi$ quasiconformal homeomorphisms $f: G \rightarrow G^{\prime}$, abbreviated $\varphi$-FQC. The definition will be given in terms of the quasihyperbolic metric. Two alternative characterizations are given: one is based on the $\theta$-mappings considered by F.W. Gehring already in 1963; the other one makes use of local quasisymmetry. Earlier results on $\theta$-mappings in normed spaces have been obtained by G. Porru $\left[\mathrm{Po}_{1}\right],\left[\mathrm{Po}_{2}\right]$ and P. Caraman [Ca].

If $E=R^{n}=E^{\prime}$, then $f$ is $\varphi$-FQC if and only if $f$ is $K$-quasiconformal in the usual sense. Here $K$ depends only on $\varphi$ and $n$, and $\varphi$ depends only on $K$. The word "free" refers to dimension-free. However, our theory differs essentially from the results called dimension-free by Anderson, Vamanamurthy and Vuorinen [AVV]. They consider $K$-quasiconformal maps in $R^{n}$ and give various estimates in terms of $K$. Their main tool is the modulus of a path family, which cannot be used in the free quasiconformal theory in the sense of the present paper. Our theory can also be called volume-free.

We assume that $E$ and $E^{\prime}$ are Banach spaces for the sake of convenience. However, the definitions are meaningful and several proofs are valid in arbitrary normed vector spaces.

I thank Pekka Alestalo, Jouni Luukkainen and Olli Martio for valuable remarks.

AMS classification: Primary 30C60, secondary 46B20. 
1.2. Restrictions. In a Banach space $E$ with $\operatorname{dim} E=\infty$ we must forgo many luxuries of $R^{n}$. These can be divided into four types:

(1) Topological. Firstly, $E$ is not locally compact and its one-point extension $\dot{E}=E \cup\{\infty\}$ is not compact. For example, we cannot use the Ascoli theorem on normal families. Furthermore, the cluster set of a map at a boundary point can be empty.

Secondly, we cannot use the pleasant topological properties of $R^{n}$ related to the Jordan-Brouwer theorem. For example, a ball $B$ in the Hilbert space $l_{2}$ is homeomorphic to the annulus $A$ between two spheres. However, it turns out that the FQC condition often prevents strange topological phenomena. In particular, there is no FQC homeomorphism between $B$ and $A$.

(2) Metric. The space $E$ is not homogeneously totally bounded (HTB) in the sense of $\left[\mathrm{TV}_{1}\right]$. This means that packing arguments usually fail in $E$.

(3) Measure-theoretic. There is no Lebesgue measure in $E$. Hence we cannot say "almost everywhere in $G \subset E$ ". The modulus of a path family, which is an important tool in the $n$-dimensional quasiconformal theory, seems to be useless in the free theory.

(4) Cubes. We can no longer use cubes or other intervals and their subdivisions. In particular, a domain has no Whitney decomposition.

1.3. What remains? The remarks in 1.2 look somewhat discouraging. However, we still have a number of useful tools. Of course, we have the norm, its triangle inequality and convexity properties. We can join points by line segments and other arcs. On the arcs we can use compactness and, in the rectifiable case, linear measure and integration. It is therefore natural that various arcs play an important role in the theory. The most useful tool turns out to be the quasihyperbolic $(\mathrm{QH})$ metric of a domain. Section 2 will be devoted to this concept.

We shall also make use of the general theory of quasisymmetric and quasimöbius maps in metric spaces. In certain problems, especially when studying the boundary behavior, the completeness of $E$ plays an essential role.

1.4. Notation and terminology. Throughout the paper $E$ and $E^{\prime}$ are Banach spaces, and $G \subset E$ and $G^{\prime} \subset E^{\prime}$ are domains. We also assume that $E \neq\{0\} \neq E^{\prime}$. The norm of a vector $x \in E$ will be written as $|x|$, the diameter of a set $A \subset E$ as $d(A)$, and the distance between nonempty sets $A, B \subset E$ as $d(A, B)$. For an open ball with center $x$ and radius $r$ we use the notation $B(x, r)$. The closed ball is $\bar{B}(x, r)$ and the boundary sphere is $S(x, r)=\partial B(x, r)$. For $x=0$ we abbreviate

$$
B(r)=B(0, r), \quad S(r)=S(0, r) .
$$

The one-point extension of $E$ is the Hausdorff space $\dot{E}=E \cup\{\infty\}$, where the neighborhoods of $\infty$ are the complements of closed bounded sets of $E$. The boundary $\partial A$ and the closure $\bar{A}$ of a set $A \subset E$ are taken in $\dot{E}$. The closed line segment with endpoints $a, b \in E$ is $[a, b]$; for a half open segment we use the 
obvious notation $[a, b)$. To simplify expressions we often omit parentheses writing $f x$ instead of $f(x)$ etc. The symbols $X, Y$ will denote metric spaces with distance written as $|a-b|$. We let $l(\gamma)$ denote the length of an arc $\gamma$. For real numbers $a, b$ we write

$$
a \vee b=\max (a, b), \quad a \wedge b=\min (a, b) .
$$

\section{Quasihyperbolic metric}

2.1. Definitions. Let $G \neq E$ be a domain. For $x \in G$ we let $\delta_{G}(x)$ denote the distance $d(x, \partial G)$. We shall usually abbreviate $\delta(x)=\delta_{G}(x), \delta^{\prime}(x)=\delta_{G^{\prime}}(x)$. If $\gamma \subset G$ is a rectifiable arc, the line integral

$$
l_{k}(\gamma)=\int_{\gamma} \frac{|d x|}{\delta(x)}
$$

is called the quasihyperbolic or QH length of $\gamma$. The quasihyperbolic distance of points $a, b \in G$ is the number

$$
k_{G}(a, b)=\inf _{\gamma} l_{k}(\gamma)
$$

over all rectifiable $\gamma$ joining $a$ and $b$ in $G$. In order to include the trivial case $a=b$, we regard a singleton $\{a\}$ as an arc of length zero. Alternatively, the arcs can be replaced by paths, which leads to the same concept. We shall usually abbreviate $k=k_{G}, k^{\prime}=k_{G^{\prime}}$. It is easy to show that $k$ is a metric in $G$ and that $l_{k}(\gamma)$ is the length of $\gamma$ in this metric. For QH balls we use the notation

$$
B_{k}(a, r)=\{x \in G: k(x, a)<r\},
$$

where $a \in G$ and $r>0$.

In a half space of $R^{n}$ the QH metric is the classical hyperbolic metric. For other domains in $R^{n}$ the $\mathrm{QH}$ metric was introduced in the seventies by Gehring and his students [GP], [GO].

Next we prove some basic inequalities between $\mathrm{QH}$ and norm distances. These will be frequently used in the paper. Most of these are well known in $R^{n}$.

2.2. Lemma. Suppose that $G \neq E$.

(1) For all $a, b \in G$ we have

$$
k(a, b) \geq \ln \left(1+\frac{|a-b|}{\delta(a)}\right) \geq \ln \frac{\delta(b)}{\delta(a)}, \quad|a-b| \leq\left(e^{k(a, b)}-1\right) \delta(a) .
$$

(2) If $a \in G, 0<t<1$ and $x, y \in \bar{B}(a, t \delta(a))$, then

$$
k(x, y) \leq \frac{1}{1-t} \frac{|x-y|}{\delta(a)} .
$$


(3) If, in addition, $t \leq \frac{1}{2}$, then

$$
k(x, y) \geq \frac{1}{1+2 t} \frac{|x-y|}{\delta(a)} .
$$

(4) If $0<r \leq \frac{1}{4}$, then $B_{k}(a, r) \subset B(a, r \delta(a) /(1-2 r))$.

Proof. Let $\gamma$ be a rectifiable arc joining $a$ and $b$ in $G$. Set $\lambda=l(\gamma)$, and let $\alpha:[0, \lambda] \rightarrow \gamma$ be the arc-length parametrization of $\gamma$. Since $\delta(\alpha(t)) \leq \delta(a)+t$, we obtain

$$
\int_{\gamma} \frac{|d x|}{\delta(x)}=\int_{0}^{\lambda} \frac{d t}{\delta(\alpha(t))} \geq \int_{0}^{\lambda} \frac{d t}{\delta(a)+t}=\ln \left(1+\frac{\lambda}{\delta(a)}\right) \geq \ln \left(1+\frac{|a-b|}{\delta(a)}\right) .
$$

This proves the first and the third inequality of (1). The second one follows from the elementary metric inequality $\delta(b) \leq \delta(a)+|a-b|$.

If $x, y \in \bar{B}(a, t \delta(a))$, then $\delta(z) \geq(1-t) \delta(a)$ for every $z \in[x, y]$. Hence (2) follows by integration along $[x, y]$. Next assume that $t \leq \frac{1}{2}$ and that $\gamma$ is a rectifiable arc joining $x$ and $y$ in $G$. If $\gamma \subset B(a, 2 t \delta(a))$, then $\delta(z) \leq(1+2 t) \delta(a)$ for every $z \in \gamma$, and hence

$$
l_{k}(\gamma) \geq \frac{|x-y|}{(1+2 t) \delta(a)}
$$

If $\gamma \not \subset B(a, 2 t \delta(a))$, then $\gamma$ has non-overlapping subarcs $\gamma_{1}, \gamma_{2}$ joining the boundary components of the annulus $A=\{u: t \delta(a)<|u-a|<2 t \delta(a)\}$ in $A$. For $z \in \gamma_{j}$ we have $\delta(z) \leq(1+2 t) \delta(a)$. Since $l\left(\gamma_{j}\right) \geq t \delta(a)$, we again obtain (2.3). This proves (3).

To prove (4) observe that $r /(1-2 r) \leq \frac{1}{2}$ for $r \leq \frac{1}{4}$. Hence (3) implies that $k(x, a) \geq r$ whenever $|x-a|=r \delta(a) /(1-2 r)$. Since $B_{k}(a, r)$ is connected, this yields (4). $\square$

2.4. Remark. For $a, b \in G$, the inner length distance $\lambda_{G}(a, b)$ is the infimum of the lengths of all arcs joining $a$ and $b$ in $G$. The proof of 2.2 shows that one can replace $|a-b|$ by $\lambda_{G}(a, b)$ in (1).

2.5. Lemma. Suppose that $a, b \in G \neq E$ and that either $|a-b| \leq \frac{1}{2} \delta(a)$ or $k(a, b) \leq 1$. Then

$$
\frac{1}{2} \frac{|a-b|}{\delta(a)} \leq k(a, b) \leq 2 \frac{|a-b|}{\delta(a)} .
$$

Hence $B_{k}(a, r) \subset B(a, 2 r \delta(a))$ for $r \leq 1$. 
Proof. If $|a-b| \leq \frac{1}{2} \delta(a)$, these inequalities follow from 2.2. If $k(a, b)=r \leq 1$, 2.2 implies

$$
\frac{|a-b|}{\delta(a)} \leq e^{r}-1 \leq 2 r,
$$

which is the first inequality. Finally, if $|a-b| \geq \frac{1}{2} \delta(a)$ and $k(a, b) \leq 1$, the second inequality holds trivially.

2.6. Lemma. For every $r>0$ there is $M=M(r) \geq 1$ such that

$$
\frac{1}{M} \frac{|x-y|}{\delta(a)} \leq k(x, y) \leq M \frac{|x-y|}{\delta(a)}
$$

for all $a \in G$ and $x, y \in \bar{B}_{k}(a, r)$.

Proof. Assume that $x, y \in \bar{B}_{k}(a, r), x \neq y$, and set

If $k(x, y) \leq 1,2.5$ and $2.2(1)$ imply

$$
p=\frac{k(x, y) \delta(a)}{|x-y|} \text {. }
$$

$$
p \leq \frac{2 \delta(a)}{\delta(x)} \leq 2 e^{r}, \quad p \geq \frac{\delta(a)}{2 \delta(x)} \geq \frac{1}{2 e^{r}} .
$$

If $k(x, y)>1,2.5$ yields $|x-y|>\frac{1}{2} \delta(x)$. Since $k(x, y) \leq 2 r$, we obtain by $2.2(1)$

$$
p \leq \frac{4 r \delta(a)}{\delta(x)} \leq 4 r e^{r}, \quad p \geq \frac{\delta(a)}{|x-a|+|a-y|} \geq \frac{1}{2 e^{r}} .
$$

2.7. Remarks. 1. The proof of 2.6 shows that we can always choose $M(r)=$ $4 e^{r}(r \vee 1)$. For $r \leq \frac{1}{4}$ we easily obtain from 2.2 the better bound $M(r)=1+4 r$.

2. The $\mathrm{QH}$ metric and the norm metric are locally bilipschitz equivalent in $G$, and hence induce the same topology in $G$. It is interesting to observe that the identity map of $B_{k}(a, r)$ from the norm metric to the $\mathrm{QH}$ metric is $\eta$ quasisymmetric with $\eta(t)=M^{2} t$ with $M=M(r)$ as in 2.6. The definition of quasisymmetry will be recalled in 3.3 .

2.8. Quasiconvexity. A metric space $X$ is $c$-quasiconvex, $c \geq 1$, if each pair $a, b$ of points in $X$ can be joined by an arc $\gamma$ with $l(\gamma) \leq c|a-b|$. In particular, an $\operatorname{arc} \gamma$ is $c$-quasiconvex if

$$
l(\gamma[x, y]) \leq c|x-y|
$$

for all $x, y \in \gamma$; here $\gamma[x, y]$ is the subarc of $\gamma$ between $x$ and $y$. In the literature this is often called the chord-arc condition.

A domain $G \neq E$ is obviously $c$-quasiconvex in the QH metric for every $c>1$. If $E=R^{n}$, it is in fact convex, that is, 1-quasiconvex. This means that each pair $a, b \in G$ can be joined by a $\mathrm{QH}$ geodesic $\gamma$ with $l_{k}(\gamma)=k(a, b)$ [GO, Lemma 1]. We show in 2.9 that this is not true in an infinite-dimensional Hilbert space. On the other hand, we shall prove in [ $\mathrm{Vä}_{4}$ ] that for every $c>1$, each pair $a, b \in G$ can be joined by an arc which is $c$-quasiconvex in the $\mathrm{QH}$ metric. 
2.9. Example. Let $E$ be a separable Hilbert space with inner product written as $x \cdot y$. Choose an orthonormal base $\left(e_{1}, e_{2}, \ldots\right)$ and a strictly increasing sequence $r_{2}, r_{3}, \ldots$ of positive numbers converging to $\frac{1}{2}$, for example, $r_{n}=(n-1) / 2 n$. Set

$$
F=S(1) \backslash \cup\left\{B\left(e_{n}, r_{n}\right): n \geq 2\right\} \text {. }
$$

We show that the points 0 and $2 e_{1}$ cannot be joined by a $\mathrm{QH}$ geodesic in the domain $G=E \backslash F$.

Let $A: E \rightarrow E$ be the isometric linear map with $A e_{1}=e_{1}, A e_{n}=e_{n+1}$ for $n \geq 2$. Then $A G \subset G$. We first show that

$$
\delta(A x) \geq \delta(x)
$$

for every $x \in G$. We may assume that $x \neq 0$. Let $y \in F=\partial G$. We must find $y^{\prime} \in F$ with $\left|y^{\prime}-x\right| \leq|y-A x|$. Set $y_{1}=A x /|A x|$ and

$$
C=\left\{z \in S(1): z \cdot e_{2}=0,\left|z-y_{1}\right| \leq\left|y-y_{1}\right|\right\}
$$

Then $C$ meets $F$, since otherwise $C$ as a connected set is contained in one of the balls $B\left(e_{n}, r_{n}\right), n \geq 3$, which easily implies $y \in B\left(e_{n}, r_{n}\right)$.

Fix a point $y_{2} \in C \cap F$. Then there is $y^{\prime} \in F$ with $A y^{\prime}=y_{2}$, and $\left|y^{\prime}-x\right|=$ $\left|y_{2}-A x\right|$. On the other hand, $\left|y_{2}-y_{1}\right| \leq\left|y-y_{1}\right|$ implies $y \cdot y_{1} \leq y_{2} \cdot y_{1}$ and thus $y \cdot A x \leq y_{2} \cdot A x$, which gives $\left|y_{2}-A x\right| \leq|y-A x|$. Hence $\left|y^{\prime}-x\right| \leq|y-A x|$, and we have proved (2.10).

Let $\gamma$ be a rectifiable arc joining 0 and $2 e_{1}$ in $G$. Then also $A \gamma$ joins these points in $G$. There is a point $z \in \gamma$ with $|z|=1$. Then clearly $\delta(A z)>\delta(z)$. Since $A$ is an isometry, this and (2.10) imply $l_{k}(A \gamma)<l_{k}(\gamma)$. Hence $\gamma$ cannot be a $\mathrm{QH}$ geodesic.

\section{Basic concepts}

3.1. Uniform continuity. A map $f: X \rightarrow Y$ is uniformly continuous if and only if there is $t_{0} \in(0, \infty]$ and an embedding $\varphi:\left[0, t_{0}\right) \rightarrow[0, \infty)$ with $\varphi(0)=0$ such that

$$
|f x-f y| \leq \varphi(|x-y|)
$$

whenever $x, y \in X$ and $|x-y|<t_{0}$. We then say that $f$ is $\left(\varphi, t_{0}\right)$-uniformly continuous. If $t_{0}=\infty$, we briefly say that $f$ is $\varphi$-uniformly continuous.

If a condition $A$ with data $v$ implies a condition $A^{\prime}$ with data $v^{\prime}$ so that $v^{\prime}$ depends only on $v$ and other given quantities, we say that $A$ implies $A^{\prime}$ quantitatively. If also $A^{\prime}$ implies $A$ quantitatively, $A$ and $A^{\prime}$ are said to be quantitatively equivalent. A symbol appearing in $A$ and $A^{\prime}$ need not have the same value in both conditions. For example, in Lemma 3.2 below, (1) with given $\varphi$ and $t_{0}$ implies (2) with $\varphi=\varphi_{1}$ depending on $\varphi, t_{0}$ and $c$. The lemma is proved in [V $\ddot{a}_{2}, 2.5$ ]. J. Luukkainen pointed out to the author that the function $\varphi_{1}$ in the proof does not necessarily satisfy the inequality $\varphi \leq \varphi_{1}$. Therefore one should redefine $\varphi_{1}(t)$ as $\varphi(t)+2\left(\varphi\left(t_{0}\right)-\varphi\left(\frac{1}{2} t_{0}\right)\right) t / t_{0}$ for $0 \leq t \leq \frac{1}{2} t_{0}$. 
3.2. Lemma. Let $X$ be $c$-quasiconvex and let $f: X \rightarrow Y$ be a map. Then the following conditions are quantitatively equivalent:

(1) $f$ is $\left(\varphi, t_{0}\right)$-uniformly continuous.

(2) $f$ is $\varphi$-uniformly continuous.

(3) $f$ is $\varphi$-uniformly continuous and there are $M \geq 0$ and $C \geq 0$ such that $\varphi(t) \leq M t+C$ for all $t$.

3.3. Definitions. We say that a homeomorphism $\varphi:[0, \infty) \rightarrow[0, \infty)$ is a growth function if $\varphi(t) \geq t$ for all $t$. If $\varphi$ is a growth function, a map $f: X \rightarrow Y$ is said to be a $\varphi$-quasi-isometry if

$$
\varphi^{-1}(|x-y|) \leq|f x-f y| \leq \varphi(|x-y|)
$$

for all $x, y \in X$. If $|f x-f y| \leq M|x-y|$ for all $x, y \in X, f$ is $M$-Lipschitz. If also $|f x-f y| \geq|x-y| / M$ and $M \geq 1, f$ is $M$-bilipschitz. This means that $f$ is a $\varphi$-quasi-isometry with $\varphi(t)=M t$. If $\eta$ is a growth function, an injective map $f: X \rightarrow Y$ is $\eta$-quasisymmetric or $\eta$-QS if $|a-x| \leq t|b-x|$ implies $|f a-f x| \leq \eta(t)|f b-f x|$ for all $x, a, b \in X$ and $t \geq 0$. A QS map is always an embedding $\left[\mathrm{TV}_{1}, 2.21\right]$.

3.4. Six classes of maps. Let $G \neq E, G^{\prime} \neq E^{\prime}$, and let $f: G \rightarrow G^{\prime}$ be a homeomorphism. We consider $G$ and $G^{\prime}$ as metric spaces with the $\mathrm{QH}$ metrics $k=k_{G}$ and $k^{\prime}=k_{G^{\prime}}$. As in $\left[\mathrm{TV}_{2}\right]$ we say that $f$ is $\varphi$-solid if it is a $\varphi$-quasiisometry in these metrics, that is,

$$
\varphi^{-1}(k(x, y)) \leq k^{\prime}(f x, f y) \leq \varphi(k(x, y))
$$

for all $x, y$ in $G$. As in $\left[\mathrm{TV}_{3}\right]$ we say that $f$ is $M$-quasihyperbolic or $M$-QH if $f$ is $M$-bilipschitz in these metrics:

$$
k(x, y) / M \leq k^{\prime}(f x, f y) \leq M k(x, y) .
$$

If $f$ is $\eta$-QS in the QH metric, we say that $f$ is $\eta$-QHQS.

For every subdomain $D \subset G, f$ defines a homeomorphism $f_{D}:\left(D, k_{D}\right) \rightarrow$ $\left(f D, k_{f D}\right)$. We say that $f$ has fully a given property if each $f_{D}$ has this property. We thus obtain the classes of fully $\varphi$-solid, fully $M$-QH and fully $\eta$-QHQS maps. These properties are also defined in the case where $G=E$ or $G^{\prime}=E^{\prime}$; the property is then required to hold for all proper subdomains $D \subset G$.

The class of fully $\varphi$-solid maps is the main object of this paper, and we shall alternatively call them freely $\varphi$-quasiconformal or $\varphi$-FQC maps.

There are obvious relations between these six classes of maps. Firstly, a fully $\varphi$-solid, fully $M$-QH or fully $\eta$-QHQS map is $\varphi$-solid, $M$-QH or $\eta$-QHQS, respectively, provided $G \neq E, G^{\prime} \neq E^{\prime}$. Furthermore, an $M$-QH map is $\varphi$-solid with $\varphi(t)=M t$ and $\eta$-QHQS with $\eta(t)=M^{2} t$, and similar implications hold for the corresponding full properties. In later sections we shall also prove that an $M$-QH map is fully $4 M^{2}-\mathrm{QH}$ (Theorem 4.7 ) and that a $\varphi$-FQC map is fully $\eta$-QHQS with some $\eta=\eta_{\varphi}$ (Theorem 5.14). 
3.5. Remark. Let us consider the special case $E=R^{n}=E^{\prime}, n \geq 2$. Then we can compare the concepts of 3.4 with the quasiconformal (QC) maps. A $K$-QC map is $\varphi-F Q C$ with

$$
\varphi(t)=C_{K}\left(t \vee t^{1 / K}\right)
$$

see [Vu, 12.20]. Conversely, a $\varphi$-FQC map is $K$-QC with $K=K(\varphi, n)$ by $\left[\mathrm{TV}_{2}\right.$, 6.12]. If $f$ is $\eta$-QHQS, it follows easily from Lemma 2.2 and from the metric definition of quasiconformality that $f$ is $K$-QC with $K=\eta(1)^{n-1}$. Conversely, a $K$-QC map is $\eta$-QHQS with $\eta=\eta_{K}$, which follows from Theorem 5.14 of the present paper.

Hence in $R^{n}$ the classes $K$-QC, $\varphi$-FQC and $\eta$-QHQS are mutually equivalent, and the quantities $K, \varphi, \eta$ depend only on each other and on $n$.

3.6. Definitions. Suppose that $f: G \rightarrow G^{\prime}$ is a homeomorphism with $G \neq E$, $G^{\prime} \neq E^{\prime}$. Let $0<t_{0} \leq 1$ and let $\theta:\left[0, t_{0}\right) \rightarrow[0, \infty)$ be an embedding with $\theta(0)=0$. We say that $f$ is $\left(\theta, t_{0}\right)$-relative if

$$
\frac{|f x-f y|}{\delta^{\prime}(f x)} \leq \theta\left(\frac{|x-y|}{\delta(x)}\right)
$$

whenever $x, y \in G$ and $|x-y|<t_{0} \delta(x)$. If $t_{0}=1$, we simply say that $f$ is $\theta$-relative. This is a well-known concept, see 3.9 .

If $f$ is $\varphi$-uniformly continuous in the QH metric, we say that $f$ is $\varphi$-semisolid. Thus $f$ is $\varphi$-solid if and only if $f$ and $f^{-1}$ are $\varphi$-semisolid.

3.7. Theorem. If $G \neq E, G^{\prime} \neq E^{\prime}$ and $f: G \rightarrow G^{\prime}$ is a homeomorphism, then the following conditions are quantitatively equivalent:

(1) $f$ is $\theta$-relative,

(2) $f$ is $\left(\theta, t_{0}\right)$-relative

(3) $f$ is $\varphi$-semisolid.

Proof. We show that (1) $\Rightarrow(2) \Rightarrow(3) \Rightarrow(1)$. The implication (1) $\Rightarrow(2)$ is trivial. Assume that (2) is true. Choose $t_{1}<t_{0}$ such that $0<t_{1} \leq \frac{1}{2}$ and $\theta\left(t_{1}\right) \leq \frac{1}{2}$. Let $x, y \in G$ with $k(x, y) \leq \frac{1}{2} t_{1}$. Then 2.5 implies $|x-y| \leq t_{1} \delta(x)$. By (2) we obtain

$$
\frac{|f x-f y|}{\delta^{\prime}(f x)} \leq \theta\left(t_{1}\right) \leq \frac{1}{2}
$$

Again by 2.5 and (2) this yields

$$
k^{\prime}(f x, f y) \leq 2 \theta(2 k(x, y))
$$

and hence $f$ is $\left(\varphi, \frac{1}{2} t_{1}\right)$-uniformly continuous in the QH metric with $\varphi(t)=2 \theta(2 t)$, and (3) follows from 3.2.

Assume that (3) holds. Suppose that $x, y \in G,|y-x|=t \delta(x), 0 \leq t<$ 1. Define the homeomorphisms $\theta_{0}:[0,1) \rightarrow[0, \infty)$ and $\psi_{0}:[0, \infty) \rightarrow[0, \infty)$ by 
$\theta_{0}(t)=t /(1-t), \psi_{0}(t)=e^{t}-1$. By $2.2(2)$ we have $k(x, y) \leq \theta_{0}(t)$, and $2.2(1)$ implies

$$
\frac{|f x-f y|}{\delta^{\prime}(f x)} \leq \psi_{0}\left(k^{\prime}(f x, f y)\right) .
$$

Hence we obtain (1) with $\theta=\psi_{0} \varphi \theta_{0}$.

3.8. Corollary. For a homeomorphism $f: G \rightarrow G^{\prime}$ the following conditions are quantitatively equivalent:

(1) $f$ and $f^{-1}$ are $\theta$-relative,

(2) $f$ and $f^{-1}$ are $\left(\theta, t_{0}\right)$-relative,

(3) $f$ is $\varphi$-solid. $\square$

3.9. Remarks. 1. Of course, the full versions of 3.7 and 3.8 are true.

2. The fully $\theta$-relative maps in $R^{n}$ were considered by Gehring [Ge, p. 14] already in 1963 . He called them briefly $\theta$-mappings and showed that this condition is equivalent to $K$-quasiconformality with $\theta$ and $K$ depending only on each other and on $n$.

3. In general normed spaces, these maps have been studied in the late seventies by G. Porru $\left[\mathrm{Po}_{1}, \mathrm{Po}_{2}\right]$ and P. Caraman [Ca].

4. By 3.2, a solid map $f: G \rightarrow G^{\prime}$ satisfies a coarse QH bilipschitz condition

$$
(k(x, y)-C) / M \leq k^{\prime}(f x, f y) \leq M k(x, y)+C .
$$

This means that $f$ is $\mathrm{QH}$ bilipschitz for large distances.

5. The relations between semisolidity and solidity are discussed in 5.12.

3.10. Compositions and inverses. Suppose that $f: G \rightarrow G^{\prime}$ and $g: G^{\prime} \rightarrow G^{\prime \prime}$ are homeomorphisms with $G^{\prime \prime} \subset E^{\prime \prime}$. It follows directly from the definitions that all six classes of 3.4 are preserved under composition. For example, if $f$ is $\varphi_{1}$-solid and $g \varphi_{2}$-solid, then $g f: G \rightarrow G^{\prime \prime}$ is $\varphi_{2} \varphi_{1}$-solid.

Similarly, the inverse $f^{-1}: G^{\prime} \rightarrow G$ of a $\varphi$-solid map $f$ is $\varphi$-solid etc. The inverse of an $\eta$-QHQS map is $\eta^{\prime}$-QHQS with $\eta^{\prime}(t)=\eta^{-1}\left(t^{-1}\right)^{-1}$.

\section{Quasihyperbolic maps}

4.1. Preliminary remarks. Recall that a homeomorphism $f: G \rightarrow G^{\prime}$ with $G \neq E, G^{\prime} \neq E^{\prime}$ is $M-\mathrm{QH}$ if it is $M$-bilipschitz in the QH metric. This class has several pleasant properties. For example, we show in 4.7 that the concepts $M-\mathrm{QH}$ and fully $M-\mathrm{QH}$ are quantitatively equivalent. From this we obtain the quantitative implications $M-\mathrm{QH} \Rightarrow \varphi$-FQC $\Rightarrow \varphi$-solid. Furthermore, the $M-\mathrm{QH}$ maps have a local characterization (see 4.6), which is useful when proving that a given map is $M-\mathrm{QH}$.

The QH maps have turned out to be useful in the QC theory of $R^{n}$. For example, the Beurling-Ahlfors extension [BA] of a QS map of $R^{1}$ to a half plane 
is QH. This property was used by Ahlfors [Ah] to obtain a bilipschitz reflection across a quasicircle through $\infty$.

If $E=E^{\prime}=R^{n}$ and $n \neq 4$, the classes $\mathrm{QH}, \mathrm{QC}$ and solid are rather close to each other. Indeed, if $f: G \rightarrow G^{\prime}$ is $\varphi$-solid and $\varepsilon>0$, there is $M=M(\varphi, \varepsilon, n) \geq$ 1 and an $M-\mathrm{QH}$ map $g: G \rightarrow G^{\prime}$ such that the QH distance $k^{\prime}(f, g)$ is less than $\varepsilon$ [TV $\left.\mathrm{TV}_{2}, 7.4\right]$. The map $g$ is also $M^{2 n-2}$-QC. In particular, two domains $G$ and $G^{\prime}$ in $R^{n}, n \neq 4$, are QH equivalent if and only if they are solidly equivalent in the natural sense.

4.2. Notation. Let $f: X \rightarrow Y$ be a map between metric spaces and let $x \in X$ be non-isolated. Then we let $L(x, f)$ and $l(x, f)$ denote the upper and lower limit, respectively, of the quotient $|f(y)-f(x)| /|y-x|$ as $y \rightarrow x$. For maps $f: G \rightarrow G^{\prime}$ we use the notation $L_{k}(x, f), l_{k}(x, f)$ when using the $\mathrm{QH}$ metric. The following lemma is obvious:

4.3. Lemma. Assume that $f: X \rightarrow Y, g: Y \rightarrow Z$ are continuous maps between metric spaces and that $x$ and $f x$ are not isolated in $X$ and $Y$, respectively. Then

$$
L(x, g f) \leq L(f x, g) L(x, f),
$$

provided that the product is not of the form $\infty \cdot 0$ or $0 \cdot \infty$. If $f: X \rightarrow Y$ is a homeomorphism, then

$$
L(x, f)=l\left(f x, f^{-1}\right)^{-1} \text {. ㄷ }
$$

4.4. Lemma. Let $f: X \rightarrow Y$ be a map. If $f$ is $M$-Lipschitz, then $L(x, f) \leq$ $M$ for all non-isolated points $x \in X$. Conversely, if $X$ is $c$-quasiconvex and if $L(x, f) \leq M$ for all $x \in X$, then $f$ is $c M$-Lipschitz.

Proof. The first assertion is obvious. The converse part is well known in the case where $X$ is a line segment [Fe, p. 64]. In the general case, let $x, y \in X$ and let $\gamma$ be an arc joining $x$ and $y$ with $l(\gamma)=\lambda \leq c|x-y|$. Let $\alpha:[0, \lambda] \rightarrow \gamma$ be the arc-length parametrization of $\gamma$. Then $\alpha$ is 1 -Lipschitz, and hence $L(t, \alpha) \leq 1$ for all $t \in[0, \lambda]$. By 4.3 we have $L(t, f \alpha) \leq M$ for all $t$. Hence the special case gives

$$
|f x-f y| \leq M \lambda \leq M c|x-y| \cdot \square
$$
Then

4.5. Lemma. Let $f: G \rightarrow G^{\prime}$ be a homeomorphism with $G \neq E, G^{\prime} \neq E^{\prime}$.

$$
L_{k}(x, f)=\frac{L(x, f) \delta(x)}{\delta^{\prime}(f x)}, \quad l_{k}(x, f)=\frac{l(x, f) \delta(x)}{\delta^{\prime}(f x)}
$$

for all $x \in G$.

Proof. This follows easily from the estimates (2) and (3) of 2.2. ㅁ 
4.6. Theorem. Let $f: G \rightarrow G^{\prime}$ be a homeomorphism with $G \neq E, G^{\prime} \neq E^{\prime}$. Then $f$ is $M$-Lipschitz in the QH metric if and only if

$$
\frac{L(x, f) \delta(x)}{\delta^{\prime}(f x)} \leq M
$$

for all $x \in G$, and $f$ is $M-\mathrm{QH}$ if and only if, in addition,

$$
\frac{L\left(f x, f^{-1}\right) \delta^{\prime}(f x)}{\delta(x)}=\frac{\delta^{\prime}(f x)}{l(x, f) \delta(x)} \leq M
$$

for all $x \in G$.

Proof. Since a domain is $c$-quasiconvex for every $c>1$ in the QH metric, the theorem follows from 4.4, 4.5 and 4.3.

4.7. Theorem. Suppose that $G \neq E, G^{\prime} \neq E^{\prime}$ and that $f: G \rightarrow G^{\prime}$ is $M-\mathrm{QH}$. Then $f$ is fully $4 M^{2}-\mathrm{QH}$.

Proof. Let $D \subset G$ be a domain. By symmetry and by 4.6 it suffices to show that

$$
\frac{\delta^{\prime}(f x) \delta_{D}(x)}{\delta(x) \delta_{f D}(f x)} \leq 4 M
$$

for all $x \in D$. By auxiliary similarities we can normalize $\delta(x)=1=\delta^{\prime}(f x)$, and the assertion reduces to

$$
\alpha(x)=\frac{\delta_{D}(x)}{\delta_{f D}(f x)} \leq 4 M .
$$

If $\delta_{f D}(f x) \geq \frac{1}{2} M$, we have $\alpha(x) \leq 2 M \delta(x)=2 M$. Assume that $\delta_{f D}(f x)<$ $1 / 2 M$. Choose $\varepsilon<1 / 2 M-\delta_{f D}(f x)$ and then $y^{\prime} \in \partial f D$ with

$$
\left|y^{\prime}-f x\right|<\delta_{f D}(f x)+\varepsilon<1 / 2 M .
$$

Then

$$
\delta^{\prime}\left(y^{\prime}\right) \geq \delta^{\prime}(f x)-1 / 2 M=1-1 / 2 M>0 .
$$

Hence $y^{\prime}=f y$ for some $y \in G \cap \partial D$. Since $f$ is $M-\mathrm{QH}$ and since $\left|f x-y^{\prime}\right|<$ $\frac{1}{2}=\frac{1}{2} \delta^{\prime}(f x), 2.5$ gives

$$
k(x, y) \leq M k^{\prime}\left(f x, y^{\prime}\right) \leq 2 M\left|f x-y^{\prime}\right| / \delta^{\prime}(f x)<1 .
$$

Again by 2.5 this implies

$$
\begin{aligned}
\delta_{D}(x) & \leq|x-y| \leq 2 k(x, y) \delta(x)=2 k(x, y) \leq 2 M k^{\prime}\left(f x, y^{\prime}\right) \leq 4 M\left|f x-y^{\prime}\right| \\
& <4 M\left(\delta_{f D}(f x)+\varepsilon\right) .
\end{aligned}
$$

As $\varepsilon \rightarrow 0$, this gives $\alpha(x) \leq 4 M$. $\square$ 
4.8. Theorem. If a homeomorphism $f: G \rightarrow G^{\prime}$ is locally $M$-bilipschitz in the norm metric, then $f$ is fully $M^{2}-\mathrm{QH}$.

Proof. Assume that $D \subset G$ is a domain with $D \neq E$. We first show that $f D \neq E^{\prime}$. Fix $a \in D$ and choose $b \in \partial D$ such that $\alpha=[a, b) \subset D$. Then $f \mid \alpha$ is $M$-Lipschitz, for example, by 4.4. Since $E^{\prime}$ is complete, $f x$ converges to a limit $b^{\prime}$ as $x \rightarrow b$ on $\alpha$. Now $b^{\prime} \notin f D$, since otherwise $b=f^{-1}\left(b^{\prime}\right) \in D$.

By 4.6 it suffices to show that $\delta_{D}(x) \leq M \delta_{f D}(f x)$ for an arbitrary $x \in D$. Let $\lambda>1$ and choose $y^{\prime} \in \partial f D$ with $\left|y^{\prime}-f x\right| \leq \lambda \delta_{f D}(f x)$. Replacing $y^{\prime}$ by a point in $\left[f x, y^{\prime}\right]$ we may assume that $\beta=\left[f x, y^{\prime}\right)$ lies in $f D$. Now $f^{-1} \mid \beta$ is $M$-Lipschitz. Arguing as above we conclude that $f^{-1}(z)$ converges to a point $y$ as $z \rightarrow y^{\prime}$ on $\beta$. Then $y \in \partial D$, since otherwise $y^{\prime}=f y \in D$. Consequently,

$$
\delta_{D}(x) \leq|y-x| \leq M\left|y^{\prime}-f x\right| \leq \lambda M \delta_{f D}(f x) .
$$

As $\lambda \rightarrow 1$, this gives the desired result. $\square$

4.9. Inversion. In the rest of this section we shall give various examples of QH maps. Let $G=E \backslash\{0\}$ and let $u: G \rightarrow G$ be the inversion $u(x)=x /|x|^{2}$. If $\operatorname{dim} E=1$, we let $G$ be one of the components of $E \backslash\{0\}$ in order that $G$ be a domain. We have

$$
|u x-u y| \leq \frac{3|x-y|}{|x||y|}
$$

for all $x, y \in G\left[\mathrm{~V} \ddot{a}_{1}, 1.6\right]$. Thus $L(x, u) \leq 3|x|^{-2}$ for all $x \in G$. Since $\delta(x)=|x|$ and $u=u^{-1}, 4.6$ implies that $u$ is $3-\mathrm{QH}$. By $4.7, u$ is fully $36-\mathrm{QH}$.

If $E$ is a Hilbert space, then $|u x-u y|=|x-y||x|^{-1}|y|^{-1}$, and $u$ is a $\mathrm{QH}$ isometry and fully $4-\mathrm{QH}$.

4.10. The radial power maps. Let $\alpha \geq 1$ and define $f: E \rightarrow E$ by $f(x)=$ $|x|^{\alpha-1} x$. Let $G$ be as in 4.9. We show that the homeomorphism $f_{1}: G \rightarrow G$ defined by $f$ is $\alpha$-QH.

We first show that $f_{1}$ is $\alpha$-Lipschitz in the QH metric. Now $\delta(x)=|x|$. By 4.6 it is sufficient to show that

$$
L(x, f) \leq \alpha|f x| /|x|=\alpha|x|^{\alpha-1}
$$

for an arbitrary $x \in G$. Let $h \in E$ with $|h|<|x|$. Then

$$
(|x|-|h|)^{\alpha-1} \leq|x+h|^{\alpha-1} \leq(|x|+|h|)^{\alpha-1} .
$$

Using this, the formula

$$
f(x+h)-f(x)=\left(|x+h|^{\alpha-1}-|x|^{\alpha-1}\right)(x+h)+|x|^{\alpha-1} h,
$$


and the derivative of the real function $u(t)=t^{\alpha-1}$ we easily obtain

$$
L(x, f) \leq(\alpha-1)|x|^{\alpha-2}|x|+|x|^{\alpha-1}=\alpha|x|^{\alpha-1} .
$$

Next observe that the inverse map $g=f_{1}^{-1}$ is of the same form $g(x)=|x|^{\beta-1} x$, but now $\beta=1 / \alpha \leq 1$. Since

$$
(|x|+|h|)^{\beta-1} \leq|x+h|^{\beta-1} \leq(|x|-|h|)^{\beta-1},
$$

an obvious modification of the preceding argument gives

$$
L(x, g) \leq(2-\beta)|x|^{\beta-1} \leq \alpha|x|^{\beta-1}=\alpha|g x| /|x| .
$$

Again by 4.6, $g$ is $\alpha$-Lipschitz in the QH metric. Hence $f_{1}$ is $\alpha$-QH. $\square$

4.11. The infinite tube. Let $E$ be a Hilbert space of dimension at least 2 with inner product written as $x \cdot y$. Let $e_{1} \in E$ be a fixed vector with $\left|e_{1}\right|=1$. The domain

$$
G=\left\{x:\left|x-\left(x \cdot e_{1}\right) e_{1}\right|<\frac{1}{2} \pi\right\}
$$

is an infinite tube with axis $\gamma=\operatorname{span} e_{1}$ and radius $\frac{1}{2} \pi$. Let $H$ be the half space $\left\{x: x \cdot e_{1}>0\right\}$. We define a homeomorphism $f: G \rightarrow H$ as follows:

Each $x \in G$ has a representation $x=s e_{1}+r y$ where $s \in R^{1}, 0 \leq r<\frac{1}{2} \pi$, $y \cdot e_{1}=0$ and $|y|=1$. The representation is unique if $r>0$. We set

$$
f(x)=e^{s}(\cos r) e_{1}+e^{s}(\sin r) y .
$$

Thus $f$ is the exponential map of the strip $0 \leq \operatorname{Im} z<\frac{1}{2} \pi$ rotated around the line $\gamma$.

We show that $f$ is $M$-QH with $M=\frac{1}{2} \pi$. Let $x=s e_{1}+r y \in G$ with $r>0$. Then $f$ has a Fréchet derivative $f^{\prime}(x)=A$ at $x$. Let $F_{1}$ be the 2-dimensional subspace of $E$ spanned by $e_{1}$ and $y$, and let $F_{2}$ be its orthogonal complement. Then $F_{1}$ and $F_{2}$ are invarint under $A$. Moreover, the maps $A_{1}=A \mid F_{1}$ and $A_{2}=A \mid F_{2}$ are linear similarities. It follows that

$$
|A|=\left|A_{1}\right| \vee\left|A_{2}\right|, \quad l(A)=\left|A_{1}\right| \wedge\left|A_{2}\right|,
$$

where now $|A|=L(x, f), l(A)=l(x, f)$. An easy computation gives $\left|A_{1}\right|=e^{s}$ and $\left|A_{2}\right|=e^{s}(\sin r) / r$. Since $r<\frac{1}{2} \pi$, we obtain $|A|=e^{s}, l(A)>2 e^{s} / \pi$. At points $x=s e_{1}$ the derivative also exists and is a similarity with $|A|=l(A)=e^{s}$. Furthermore, setting $t=\frac{1}{2} \pi-r$ we have

$$
\delta(x)=t, \quad \delta^{\prime}(f x)=e^{s} \sin t,
$$

and hence

$$
\frac{L(x, f) \delta(x)}{\delta^{\prime}(f x)}=\frac{t}{\sin t}<\frac{1}{2} \pi, \quad \frac{l(x, f) \delta(x)}{\delta^{\prime}(f x)}>\frac{2 t}{\pi \sin t}>\frac{2}{\pi} .
$$

By $4.6, f$ is $\frac{1}{2} \pi-\mathrm{QH}$.

Observe that the argument is the same in finite- and infinite-dimensional spaces. The next example illustrates a new phenomenon which does not occur in $R^{n}$. 
4.12. The broken tube. Let $E$ be an infinite-dimensional separable Hilbert space. Choose an orthonormal base $\left(e_{j}\right)_{j \in \mathbf{Z}}$ of $E$ indexed by the set $\mathbf{Z}$ of all integers. Setting $\gamma_{j}^{\prime}=\left[e_{j-1}, e_{j}\right]$ we obtain the infinite broken line

$$
\gamma^{\prime}=\bigcup\left\{\gamma_{j}^{\prime}: j \in \mathbf{Z}\right\} .
$$

As in 4.11 we let $\gamma$ denote the line spanned by $e_{1}$. We divide $\gamma$ to line segments $\gamma_{j}=\left[a_{j-1}, a_{j}\right]$ with $a_{j}=j \sqrt{2} e_{1}$. Then there is a natural homeomorphism $f_{0}: \gamma \rightarrow$ $\gamma^{\prime}$ which maps each $\gamma_{j}$ isometrically onto $\gamma_{j}^{\prime}$. It is rather obvious that for small $r$, say for $r \leq 1 / 10$, we can extend $f_{0}$ to a homeomorphism $f$ of the tube $G=$ $\gamma+B(r)$ onto a neighborhood $G^{\prime}$ of $\gamma^{\prime}$ such that $f$ is locally $M$-bilipschitz with a universal $M$. The domain $G^{\prime}$ is a broken tube. By $4.8 f$ is $M^{2}-\mathrm{QH}$. Using the map of 4.11 and a similarity map we obtain a $\pi M^{2} / 2-\mathrm{QH}$ map $g: H \rightarrow G^{\prime}$. The cluster sets of $g$ at 0 and at $\infty$ are empty.

We conclude this section by some well-known inequalities and bilipschitz constructions which will be needed in Section 5 .

4.13. Lemma. For every pair $x, y \in E \backslash\{0\}$ and for $p x=x /|x|$ we have $|p x-p y| \leq 2|x-y| /|x|$.

Proof. $|x||p x-p y| \leq|x-| x|y /| y|| \leq|x-y|+|| x|-| y|| \leq 2|x-y|$. व

4.14. Radial bilipschitz maps. Suppose that $g:\left[0, r_{1}\right] \rightarrow\left[0, r_{2}\right]$ is an increasing $M$-bilipschiz homeomorphism. Write again $p(x)=x /|x|$ for $x \in E \backslash\{0\}$. Setting

$$
f(x)=g(|x|) p(x), \quad f(0)=0
$$

we obtain a homeomorphism $f: B\left(r_{1}\right) \rightarrow B\left(r_{2}\right)$. The possibility $r_{1}=r_{2}=\infty$ is allowed; then $f: E \rightarrow E$. Since $g(t) \leq M t$, Lemma 4.13 implies for all $x, y \in$ $B\left(r_{1}\right) \backslash\{0\}$

$$
|f x-f y| \leq g(|x|)|p x-p y|+|g(|x|)-g(|y|)| \leq 3 M|x-y| .
$$

By symmetry and continuity, $f$ is $3 M$-bilipschitz.

\section{Free quasiconformality}

5.1. Summary. In this section we mainly consider various quasisymmetry properties of FQC maps. We also show that these maps are Hölder continuous and that the distortion function $\varphi$ of an FQC map can always be chosen to be of the form $\varphi(t)=C\left(t^{\alpha} \vee t\right), \alpha \leq 1$.

5.2. Weak quasisymmetry. For $H \geq 1$, an embedding $f: X \rightarrow Y$ is weakly $H$-quasisymmetric if $|a-x| \leq|b-x|$ implies $|f a-f x| \leq H|f b-f x|$ for all $a, b, x \in X$. Thus an $\eta$-QS map is weakly $H$-QS with $H=\eta(1)$. The converse result is true in some special cases. For example, if $X \subset R^{n}$ is pathwise connected and if $f: X \rightarrow R^{n}$ is weakly $H$-QS, then $f$ is $\eta$-QS with $\eta=\eta(H, n)$; see $\left[\mathrm{Vä}_{3}, 2.9\right]$. The corresponding statement in arbitrary Banach spaces is not true. However, we shall show that the ideas of $\left[\mathrm{Vä}_{3}, 2.9\right]$ can be used in the case where $X$ and $f X$ are quasiconvex. 
5.3. Lemma. Suppose that $X$ is c-quasiconvex, that $f: X \rightarrow Y$ is weakly $H$-QS and that $a, b, x \in X$ with $|a-x|=t|b-x|$. Then

$$
|f a-f x| \leq \eta(t)|f b-f x|
$$

where $\eta:[0, \infty) \rightarrow[0, \infty)$ is an increasing function depending only on $H$ and $c$.

Remark. Since $\eta(t)$ is not required to tend to zero as $t \rightarrow 0, f$ is not necessarily QS.

Proof. For $t \leq 1$ we can choose $\eta(t)=H$. Assume that $t>1$. By $\left[\mathrm{TV}_{1}\right.$, $2.12]$ it suffices to show that $X$ is $C$-pseudoconvex in the sense of $\left[\mathrm{TV}_{1}, 2.7\right]$ with $C$ depending on $c$.

Let $a, b \in X$ and suppose $0<r \leq|a-b|$. Join $a$ and $b$ by an arc $\gamma$ with $l(\gamma) \leq c|a-b|$. Set $a_{0}=a$ and choose inductively successive points $a_{1}, \ldots, a_{s}=b$ of $\gamma$ such that $a_{j+1}$ is the last point of $\gamma$ in $\bar{B}\left(a_{j}, r\right)$. Then $\left|a_{j}-a_{j-1}\right|=r$ for $1 \leq j \leq s-1$ and $\left|a_{s}-a_{s-1}\right| \leq r$. Hence $(s-1) r \leq l(\gamma) \leq c|a-b|$. Thus $X$ is $C$-pseudoconvex with $C(t)=1+c t$. -

5.4. Lemma. Suppose that $f: X \rightarrow Y$ is weakly $H-Q S$ and that $f X$ is $c$-quasiconvex. If $x, a, b \in X$ with $|a-x|=t|b-x|$ and if $0 \leq t \leq 1$, then

$$
|f a-f x| \leq \eta(t)|f b-f x|,
$$

where $\eta:[0,1] \rightarrow[0, \infty)$ is an embedding with $\eta(0)=0$ depending only on $H$ and $c$.

Proof. Since the inequality is true with $\eta(t)=H$, we may assume that $0<t \leq \frac{1}{4}$. Join $f b$ and $f x$ by an arc $\gamma \subset f X$ with $l(\gamma) \leq c|f b-f x|$. Choose successive points $b=b_{0}, b_{1}, \ldots, b_{s}$ of $f^{-1} \gamma$ such that $\left|b_{j}-x\right|=3^{-j}|b-x|$ and $s$ is the least integer with $3^{-s} \leq t$. Then

$$
s \geq \frac{\ln (1 / t)}{\ln 3}=s_{0}(t) \rightarrow \infty
$$

as $t \rightarrow 0$. Since $t \leq \frac{1}{4}$, we have $s \geq 2$.

For $1 \leq j \leq s-1$ we have

$$
\left|b_{j}-a\right| \leq\left|b_{j}-x\right|+|x-a| \leq 2\left|b_{j}-x\right| \leq\left|b_{j}-b_{j-1}\right| .
$$

Moreover, $\left|b_{j}-x\right| \leq\left|b_{j}-b_{j-1}\right|$. Since $f$ is weakly $H$-QS, we obtain

$$
|f a-f x| \leq\left|f a-f b_{j}\right|+\left|f b_{j}-f x\right| \leq 2 H\left|f b_{j}-f b_{j-1}\right|
$$

Summing over $1 \leq j \leq s-1$ yields

$$
(s-1)|f a-f x| \leq 2 H l(\gamma) \leq 2 H c|f b-f x| .
$$

Hence we can choose

$$
\eta(t)=\frac{2 H c}{s_{0}(t)-1}
$$

5.5. Theorem. Suppose that $f: X \rightarrow Y$ is weakly $H-\mathrm{QS}$ and that $X$ and $f X$ are $c$-quasiconvex. Then $f$ is $\eta$-QS with $\eta$ depending only on $H$ and $c$. 
Proof. This follows directly from 5.3 and 5.4. $\square$

5.6. Definition. Let $0<q<1$. A homeomorphism $f: G \rightarrow G^{\prime}$ is $q$-locally $\eta$-quasisymmetric if $f \mid B(a, q r)$ is $\eta$-QS whenever $B(a, r) \subset G$. If $G \neq E$, this means that $f \mid B(a, q \delta(a))$ is $\eta$-QS. If $G=E$, this means that $f$ is $\eta$-QS.

A $K$-QC map in $R^{n}$ is $q$-locally $\eta$-QS for every $q<1$ with $\eta$ depending on $K$ and $q$ [AVV, 5.23]. Conversely, a $q$-locally $\eta$-QS map in $R^{n}$ is $K$-QC with $K=\eta(1)^{n-1}$ by the metric definition of quasiconformality.

We shall next prove the corresponding free results.

5.7. Theorem. Suppose that $f: G \rightarrow G^{\prime}$ is fully $\varphi$-semisolid and that $0<q<1$. Then $f$ is $q$-locally $\eta$-QS with $\eta$ depending only on $\varphi$ and $q$.

Proof. We first show that $f$ is $1 / 3$-locally $\eta$-QS with $\eta=\eta_{\varphi}$. Let $x_{0} \in G$ and assume $B\left(x_{0}, r\right) \subset G$. Let $x, a, b \in B=B\left(x_{0}, r / 3\right)$ with $|a-x|=t|b-x|$. We must find an estimate

$$
|f a-f x| \leq \eta(t)|f b-f x|
$$

where $\eta(t) \rightarrow 0$ as $t \rightarrow 0$. We consider three cases.

Case 1. $t \leq 2 / 3$. By the full version of $3.7, f$ is fully $\theta$-relative with some $\theta=\theta_{\varphi}$. We apply relativity in the domain $D=G \backslash\{b\}$. If $\operatorname{dim} E=1$, we let $D$ be the $x$-component of $G \backslash\{b\}$. We have $\delta_{D}(x)=|x-b|$ and hence $|x-a|=t \delta_{D}(x)$. Thus

$$
|f a-f x| \leq \theta(t) d(f x, \partial f D) .
$$

Since $d(f x, \partial f D) \leq|f b-f x|$, we can choose $\eta(t)=\theta(t)$, which has the desired behavior as $t \rightarrow 0$.

Case 2. $2 / 3<t \leq 1$. Writing $z=2 a / 3+x / 3$ and applying Case 1 twice we get

$$
\begin{aligned}
|f a-f x| & \leq|f a-f z|+|f z-f x| \\
& \leq\left(\theta\left(\frac{1}{2}\right)+1\right)|f z-f x| \\
& \leq\left(\theta\left(\frac{1}{2}\right)+1\right) \theta\left(\frac{2}{3}\right)|f b-f x| .
\end{aligned}
$$

Case 3. $t>1$. From the preceding cases it follows that $f \mid B$ is weakly $H$-QS with $H=H(\varphi)$. Since $B$ is convex, the desired estimate follows from 5.3.

Next assume that $1 / 3<q<1$. We shall reduce this case to the case $q=1 / 3$ by an auxiliary map. Let $g:[0,1] \rightarrow[0,1]$ be the increasing homeomorphism with $g(1 / 3)=q$ which is affine on $[0,1 / 3]$ and on $[1 / 3,1]$. Then $g$ is $M$-bilipschitz with $M=M(q)$. Suppose that $B\left(x_{0}, r\right) \subset G$. Applying 4.14 and auxiliary similarities we obtain a radial $3 M$-bilipschitz homeomorphism $h_{0}: B\left(x_{0}, r\right) \rightarrow B\left(x_{0}, r\right)$ which maps $B\left(x_{0}, r / 3\right)$ onto $B\left(x_{0}, q r\right)$. Moreover, $h_{0}$ can be extended by identity to a homeomorphism $h: E \rightarrow E$, which is also $3 M$-bilipschitz. By $4.8 h$ defines a fully $9 M^{2}-\mathrm{QH}$ map $h_{1}: G \rightarrow G$. Then the homeomorphism $f_{1}=f h_{1}: G \rightarrow G^{\prime}$ 
is fully $\varphi_{1}$-semisolid with $\varphi_{1}(t)=\varphi\left(9 M^{2} t\right)$. Hence $f_{1} \mid B\left(x_{0}, r / 3\right)$ is $\eta$-QS with $\eta=\eta(\varphi, q)$. Since $h \mid B\left(x_{0}, r / 3\right)$ is a similarity map onto $B\left(x_{0}, q r\right), f \mid B\left(x_{0}, q r\right)$ is $\eta$-QS. $\square$

5.8. Remark. Related results were proved by G. Porru in the late seventies. In particular, Theorem 2.1 of $\left[\mathrm{PO}_{2}\right]$ states that a fully $\theta$-relative map is locally weakly QS.

5.9. Theorem. If a homeomorphism $f: G \rightarrow G^{\prime}$ is $q$-locally $\eta$-QS, then $f$ is fully $\varphi$-semisolid with $\varphi$ depending on $\eta$ and $q$.

Proof. Let $D \subset G$ be a domain with $D \neq E, f D \neq E^{\prime}$. Then $f$ defines a $q$-locally $\eta$-QS homeomorphism $f_{1}: D \rightarrow f D$. By 3.7 it suffices to show that $f_{1}$ is $(\theta, q)$-relative with $\theta(t)=\eta(t / q)$.

Suppose that $a \in D$ and $b \in B\left(a, q \delta_{D}(a)\right)=B$. Let $\lambda>1$ and choose a point $z \in \partial f D$ with $|z-f a| \leq \lambda \delta_{f D}(f a)$. Let $y$ be the first point of $[f a, z]$ which is not in $f B$. Since $f$ is $\eta$-QS in $\bar{B}$ and since $\bar{B}$ is complete, $f \bar{B}$ is complete by $\left[\mathrm{TV}_{1}, 2.24\right]$, and hence $y \in f \bar{B}$. Writing $x=f^{-1}(y)$ we have $|x-a|=q \delta_{D}(a)$, and thus

$$
\frac{|f b-f a|}{\delta_{f D}(f a)} \leq \lambda \frac{|f b-f a|}{|y-f a|} \leq \lambda \eta\left(\frac{|b-a|}{|x-a|}\right)=\lambda \eta\left(\frac{|b-a|}{q \delta_{D}(a)}\right) .
$$

Since $\lambda>1$ is arbitrary, this proves the $(\theta, q)$-relativity of $f_{1}$. व

5.10. Theorem. For a homeomorphism $f: G \rightarrow G^{\prime}$ the following conditions are quantitatively equivalent:

(1) $f$ is $\varphi$-FQC.

(2) $f$ and $f^{-1}$ are $q$-locally $\eta$-QS.

(3) For every $q \in(0,1)$ there is $\eta_{q}$ such that $f$ and $f^{-1}$ are $q$-locally $\eta_{q}$-QS.

Proof. Observe that the data for (1) is $\varphi$, for (2) the pair $(q, \eta)$ and for (3) the function $q \mapsto \eta_{q}$. The implication (3) $\Rightarrow(2)$ is trivial, (2) $\Rightarrow(1)$ follows from 5.9 , and $(1) \Rightarrow(3)$ from 5.7 .

5.11. Summary. We have three characterizations for free quasiconformality: (1) full solidity, (2) full relativity of $f$ and $f^{-1},(3) q$-local quasisymmetry of $f$ and $f^{-1}$. These are given in the original definition 3.4 and in Theorems 3.8 (cf. 3.9.1) and 5.10.

We also remark that the proof of 5.7 in the case $\operatorname{dim} E \geq 2$ made only use of domains $D=G \backslash\{a\}, a \in G$. Consequently, if $f$ is $\varphi$-solid for every such $D, f$ is $\psi$-FQC with $\psi=\psi_{\varphi}$.

5.12. One-sided conditions. It is natural to ask whether a fully $\varphi$-semisolid map $f: G \rightarrow G^{\prime}$ is $\psi-\mathrm{FQC}$ with $\psi=\psi_{\varphi}$. In the one-dimensional case this is not true, see 6.8. We do not know the answer in higher dimensions. However, 
if $E=E^{\prime}=R^{n}, f$ is $\psi$-FQC with $\psi=\psi(\varphi, n)$. Indeed, by 3.7 and by [Ge, Theorem 4], full $\varphi$-semisolidity is equivalent to $K$-quasiconformality with $\varphi$ and $K$ depending on each other and on $n$.

We next prove that the answer is positive if $G$ is the whole space $E$. We also show that $f E=E^{\prime}$.

5.13. Theorem. Suppose that $f: E \rightarrow G^{\prime} \subset E^{\prime}$ is fully $\varphi$-semisolid. Then

(1) $G^{\prime}=E^{\prime}$

(2) $f$ is $\eta$-QS with $\eta=\eta_{\varphi}$,

(3) $f$ is $\psi$-FQC with $\psi=\psi_{\varphi}$.

Proof. By 5.7, $f$ is $\frac{1}{2}$-locally $\eta$-QS with $\eta=\eta_{\varphi}$. Since the domain $G$ is now $E, f$ is $\eta$-QS in $E$. Since QS maps preserve completeness, $G^{\prime}$ is closed in $E^{\prime}$, and hence $G^{\prime}=E^{\prime}$. Since $f^{-1}: E^{\prime} \rightarrow E$ is $\eta^{\prime}$-QS with $\eta^{\prime}(t)=\eta^{-1}\left(t^{-1}\right)^{-1}$, (3) follows from 5.10. व

5.14. Theorem. A $\varphi$-FQC map $f: G \rightarrow G^{\prime}$ is fully $\eta$-QHQS with $\eta=\eta_{\varphi}$.

Proof. We may assume that $G \neq E, G^{\prime} \neq E^{\prime}$. It suffices to show that $f$ is $\eta$-QHQS. Since $G$ and $G^{\prime}$ are $c$-quasiconvex in the QH metric for all $c>1$, it suffices to show, by 5.5, that $f$ is weakly H-QS in the QH metric with $H=H(\varphi)$.

Suppose that $x, a, b \in G$ with $k(a, x) \leq k(b, x)=r>0$. We must find an upper bound $\rho \leq H(\varphi)$ for the ratio

$$
\rho=\frac{k^{\prime}(f a, f x)}{k^{\prime}(f b, f x)}
$$

By $5.10, f$ is $1 / 3$-locally $\eta$-QS with $\eta=\eta_{\varphi}$. By 2.5 , we have $B_{k}(x, 1 / 6) \subset$ $B(x, \delta(x) / 3)$. Set $r_{0}=(1 / 6) \wedge \varphi^{-1}(1)$. Then $f$ is $\eta$-QS in $B_{k}\left(x, r_{0}\right)$, and $f B_{k}\left(x, r_{0}\right) \subset B_{k^{\prime}}(f x, 1)$.

By 3.2 we may assume that there are $M$ and $C$ such that $\varphi(t) \leq M t+C$ for all $t \geq 0$. We consider three cases.

Case 1. $r<r_{0}$. Now 2.5 yields

$$
\rho \leq \frac{4|f a-f x|}{|f b-f x|} \leq 4 \eta\left(\frac{4 k(a, x)}{k(b, x)}\right) \leq 4 \eta(4) .
$$

Case 2. $r_{0} \leq r<2 C$. Now $\rho \leq \varphi(2 C) / \varphi^{-1}\left(r_{0}\right)$.

Case 3. $r \geq 2 C$. Now $\varphi^{-1}(r) \geq(r-C) / M \geq r / 2 M$, and hence

$$
\rho \leq \frac{M r+C}{r / 2 M} \leq 2 M^{2}+M \text {. }
$$

5.15. Theorem. Suppose that $f: G \rightarrow G^{\prime}$ is $\varphi$-FQC, $G \neq E$. In each $\mathrm{QH}$ ball $B_{k}(z, r), f$ is $\eta$-QS with $\eta$ depending on $\varphi$ and $r$. 
Proof. By 5.14, $f$ is $\eta$-QHQS with $\eta=\eta_{\varphi}$. Let $M=M(\varphi(r))$ be the number given by Lemma 2.6. If $x, a, b \in B_{k}(z, r)$ with $|a-x| \leq t|b-x|$, then the images of these points are in $B_{k^{\prime}}(f z, \varphi(r))$, and 2.6 easily implies

$$
|f a-f x| \leq M^{2} \eta\left(M^{2} t\right)|f b-f x| .
$$

Hence $f$ is $\eta_{1}$-QS in $B_{k}(z, r)$ with $\eta_{1}(t)=M^{2} \eta\left(M^{2} t\right)$. 口

5.16. Remark. For QC maps in $R^{n}$, Theorem 5.15 is an unpublished result of O. Martio.

5.17. Quasimöbius maps. Let $\eta$ be a growth function. An injective map $f: X \rightarrow Y$ is called $\eta$-quasimöbius or $\eta$-QM if each cross ratio

$$
\tau=\frac{|a-b||c-d|}{|a-c||b-d|}
$$

of distinct points $a, b, c, d$ in $X$ satisfies the inequality $f \tau \leq \eta(\tau)$ where $f \tau$ is the cross ratio of the points $f a, f b, f c, f d$. The basic QM theory is given in [Vä1]. An $\eta$-QS map is $\eta_{1}$-QM with $\eta_{1}=\eta_{1}(\eta)$. Roughly speaking, the essential difference between QS and QM maps is that a QM map need not fix the point at infinity. However, we do not consider in this paper domains which contain $\infty$.

In $R^{n}$ an $\eta$-QM homeomorphism $f: G \rightarrow G^{\prime}$ is $K$-QC with $K=\eta(1)^{n-1}$. We next prove the corresponding free result:

5.18. Theorem. If $f: G \rightarrow G^{\prime}$ is $\eta$-QM, $f$ is $\varphi$-FQC with $\varphi=\varphi_{\eta}$.

Proof. Suppose that $D \subset G$ is a domain with $D \neq E, f D \neq E^{\prime}$. We apply [V $\left.\ddot{a}_{1}, 3.19\right]$ observing the misprint $f A \backslash\{\infty\}$ which should be $\overline{f A} \backslash\{\infty\}$. Thus $f$ has a homeomorphic extension $\bar{f}: \bar{D} \rightarrow \overline{f D}$. By auxiliary translations we may assume that $0 \in \partial D$ and that either $\bar{f}(0)=0$ or $\bar{f}(0)=\infty$. Let $u$ be the inversion $u(x)=x /|x|^{2}$. If $\bar{f}(0)=0$, we define $g: u D \rightarrow u f D$ by $g(x)=u f u(x)$. If $\bar{f}(0)=\infty$, we define $g: u D \rightarrow f D$ by $g(x)=f u(x)$. In both cases $g$ is $\eta_{1}$-QM with $\eta_{1}$ depending on $\eta$; see [V $\left.\ddot{a}_{1}, 1.6\right]$. Since $g(x) \rightarrow \infty$ as $x \rightarrow \infty, g$ is $\eta_{1}$-QS. By $5.10, g$ is $\varphi_{1}-\mathrm{FQC}$ with $\varphi_{1}=\varphi_{1}(\eta)$. By $4.9, u$ is fully $36-\mathrm{QH}$, and hence $f$ is $\varphi$-FQC with $\varphi=\varphi_{\eta}$. व

5.19. Theorem. Suppose that $f: G \rightarrow G^{\prime}$ is $\varphi$-FQC with $G \neq E, G^{\prime} \neq E^{\prime}$. Then $f$ satisfies the Hölder condition

$$
\frac{|f a-f b|}{\delta^{\prime}(f a)} \leq C\left(\frac{|a-b|}{\delta(a)}\right)^{\alpha}
$$

for all $a \in G$ and $b \in \bar{B}\left(a, \frac{1}{2} \delta(a)\right)$. The constants $C \geq 1$ and $\alpha \leq 1$ depend only on $\varphi$. 
Proof. By 5.10, $f$ is $\frac{1}{2}$-locally $\eta$-QS with $\eta=\eta_{\varphi}$. Fix $a \in G$ and define a homeomorphism $g: \bar{B}\left(\frac{1}{2}\right) \rightarrow Q \subset E^{\prime}$ by

$$
g(x)=\frac{f(a+\delta(a) x)}{\delta^{\prime}(f a)} .
$$

Then $g$ is $\eta$-QS. By $\left[\mathrm{TV}_{1}, 3.14\right], g$ satisfies a Hölder condition

$$
|g(x)-g(0)| \leq c|x|^{\alpha}
$$

with $\alpha \leq 1$ and $c \geq 1$ depending on $\varphi$ and on the numbers $d(Q)$ and $d\left(B\left(\frac{1}{2}\right)\right)$ $=1$. If $|a-b|<\frac{1}{2} \delta(a)$, then $k(a, b)<1$ by 2.5 . Hence $k^{\prime}(f a, f b)<\varphi(1)$. By 2.2 (1) this implies $|f a-f b|<\delta^{\prime}(f a) e^{\varphi(1)}$, and hence $d(Q) \leq 2 e^{\varphi(1)}$. Hence $c$ and $\alpha$ depend only on $\varphi$. Since

$$
f(z)=\delta^{\prime}(f a) g\left(\frac{z-a}{\delta(a)}\right)
$$

for $z \in \bar{B}\left(a, \frac{1}{2} \delta(a)\right)$, we obtain

$$
\frac{|f a-f b|}{\delta^{\prime}(f a)}=\left|g(0)-g\left(\frac{b-a}{\delta(a)}\right)\right| \leq c\left(\frac{|a-b|}{\delta(a)}\right)^{\alpha} \cdot \text {. }
$$

5.20. Theorem. If $f: G \rightarrow G^{\prime}$ is $\varphi$-FQC, then $f$ is $\varphi_{1}$-FQC with some $\varphi_{1}$ of the form $\varphi_{1}(t)=C\left(t^{\alpha} \vee t\right)$, where $C \geq 1$ and $\alpha \leq 1$ depend only on $\varphi$.

Proof. Let $D \subset G$ be a domain with $D \neq E, f D \neq E^{\prime}$. Let $C$ and $\alpha$ be the numbers given by 5.19. Set $t_{0}=\frac{1}{4} \wedge \varphi^{-1}(1)$. If $x, y \in D$ and $k_{D}(x, y) \leq t_{0}$, then 2.5 gives $|x-y| \leq \frac{1}{2} \delta_{D}(x)$, and by 5.19 we have

$$
\frac{|f x-f y|}{\delta_{f D}(f x)} \leq C\left(\frac{|x-y|}{\delta_{D}(x)}\right)^{\alpha} .
$$

Since $k_{f D}(f x, f y) \leq 1$, this and 2.5 yield

$$
k_{f D}(f x, f y) \leq 2 C\left(2 k_{D}(x, y)\right)^{\alpha} .
$$

Hence we can choose $\varphi_{1}(t)=2^{1+\alpha} C t^{\alpha}$ for $t \leq t_{0}$.

Next assume that $k_{D}(x, y)=t \geq t_{0}$. By 3.2 , we may assume that $\varphi(t) \leq$ $M t+C_{1}$ for some $M \geq 1$ and $C_{1} \geq 0$ depending on $\varphi$. Hence

$$
k_{f D}(f x, f y) \leq M t+C_{1} \leq\left(M+C_{1} / t_{0}\right) t=C_{2} t .
$$

Hence we can choose $\varphi_{1}(t)=C_{2} t$ for $t \geq t_{0}$. 


\section{Finite-dimensional spaces}

6.1. Preliminary remarks. In this section we briefly consider the case where $\operatorname{dim} E=\operatorname{dim} E^{\prime}=n<\infty$. If the norms of $E$ and $E^{\prime}$ are induced by an inner product, we can identify $E=R^{n}=E^{\prime}$. In this case, assuming $n \geq 2$, the free $\varphi$-quasiconformality is equivalent to ordinary $K$-quasiconformality, as explained in 3.5. The case $n=1$ will be considered in 6.7.

If $E$ is a Banach space of dimension $n$, there is a $\sqrt{n}$-bilipschitz linear map $T: E \rightarrow R^{n}$ by a classical result of Banach space theory, recalled in 6.2 below. This reduces the $n$-dimensional FQC theory to the case $E=R^{n}=E^{\prime}$, as far as we are not interested in sharp quantitative results or the special features of $\varphi$-FQC maps with $\varphi$ close to the identity.

6.2. Lemma. Suppose that $\operatorname{dim} E=\operatorname{dim} E^{\prime}=n$. Then there is a linear map $T: E \rightarrow E^{\prime}$ such that

$$
|x| \leq|T x| \leq n|x|
$$

for all $x \in E$. Thus $T$ is $n$-bilipschitz. There is also a homeomorphism $h: E \rightarrow E^{\prime}$ such that

(1) $h(\lambda x)=\lambda h(x)$ for all $x \in E$ and $\lambda \in R^{1}$,

(2) $|h x|=|x|$ for all $x \in E$,

(3) $h$ is $M$-bilipschitz with $M=2 n+1$.

Proof. The first part of the lemma was proved by F. John in 1948, see [MS, 3.3]. The second part follows from this with

$$
h x=\frac{|x| T x}{|T x|}
$$

for $x \neq 0$. The properties (1) and (2) are clear, and (3) follows by elementary estimates with 4.13. $\square$

6.3. Applications. Suppose that $\operatorname{dim} E=\operatorname{dim} E^{\prime}=n$. We first observe that any linear bijection $T: E \rightarrow R^{n}$ is bilipschitz, and hence the qualitative results of the QC theory of $R^{n}$ are readily extended to FQC maps between domains in $E$ and $E^{\prime}$. For example, an FQC map $f: G \rightarrow G^{\prime}$ is ACL and a.e. differentiable; in $E$ we use any translation-invariant Haar measure.

For quantitative results we use 6.2. As an example we give the local metric definition for $\mathrm{FQC}$ maps. We let $H(x, f)$ denote the metric (or linear) dilatation of $f$ at $x$; see e.g. [V $\ddot{a}_{1}$, p. 231].

6.4. Theorem. Suppose that $\operatorname{dim} E=\operatorname{dim} E^{\prime}=n, 2 \leq n<\infty$, and that $f: G \rightarrow G^{\prime}$ is $\varphi$-FQC. Then $H(x, f) \leq H_{0}=H_{0}(\varphi)$ for all $x \in G$. Conversely, if $f$ is a homeomorphism with $H(x, f) \leq H_{0}$ for all $x \in G$, then $f$ is $\varphi$-FQC with $\varphi$ depending on $H_{0}$ and $n$. 
Proof. Suppose that $f$ is $\varphi$-FQC. By $5.10, f$ is $\frac{1}{2}$-locally $\eta_{\varphi}$-QS. Hence $H(x, f) \leq \eta_{\varphi}(1)=H_{0}(\varphi)$. This part is valid without dimensional restrictions.

Next suppose that $H(x, f) \leq H_{0}$ for all $x \in G$. Choose linear maps $T: E \rightarrow$ $R^{n}, T^{\prime}: E^{\prime} \rightarrow R^{n}$ as in 6.2. Setting $f_{1}(x)=T^{\prime} f T^{-1}(x)$ we obtain a homeomorphism $f_{1}: T G \rightarrow T^{\prime} G^{\prime}$. With the customary notation [V $\ddot{a}_{1}$, p. 231] we have

$$
\limsup _{r \rightarrow 0} \frac{L\left(x, f_{1}, r / n\right)}{l\left(x, f_{1}, r\right)} \leq n H_{0}
$$

for all $x \in T G$. By [Cr, Theorem 1] this implies that $f_{1}$ is $K$-QC with $K=$ $K\left(H_{0}, n\right)$. Hence $f_{1}$ is $\varphi_{1}$-FQC with $\varphi_{1}=\varphi_{1}\left(H_{0}, n\right)$, see 3.5. Since $T$ and $T^{\prime}$ are $n$-bilipschitz, the theorem follows by 4.8 . $\square$

6.5. The radial power map. Theorem 6.2 can sometimes be also applied in infinite-dimensional spaces. Let $E$ be a Banach space, let $\alpha \geq 1$, and define $f: E \rightarrow E$ by $f(x)=|x|^{\alpha-1} x$. This map is $\eta$-QS with $\eta=\eta_{\alpha}$, but a direct proof seems awkward. However, since each triple is contained in a linear subspace $E_{0}$ with $\operatorname{dim} E_{0} \leq 3$ and since $f E_{0}=E_{0}$, we may assume that $\operatorname{dim} E=\operatorname{dim} E^{\prime}=3$. Using the map $h$ of 6.2 we reduce the problem to the case $f: R^{3} \rightarrow R^{3}$, where the result follows from the quasiconformality of $f$.

An alternative proof is based on 4.10. Indeed, in the domain $E \backslash\{0\} f$ is $\alpha-\mathrm{QH}$ and hence $\varphi_{\alpha}$-FQC. By a removability result in [V $\left.\ddot{a}_{4}\right], f$ is $\psi_{\alpha}$-FQC in $E$ and hence $\eta_{\alpha}$-QS by 5.13 .

6.6. The case $n=1$. Suppose that $\operatorname{dim} E=\operatorname{dim} E^{\prime}=1$. Then we can identify $E=R^{1}=E^{\prime}$. A domain in $R^{1}$ is an open interval $(a, b)$, where possibly $a=-\infty$ or $b=\infty$. The following result identifies the FQC maps in $R^{1}$ :

6.7. Theorem. Suppose that $G$ and $G^{\prime}$ are domains in $R^{1}$ and that $f: G \rightarrow G^{\prime}$ is a homeomorphism. Then the following conditions are quantitatively equivalent:

(1) $f$ is $\varphi$-FQC,

(2) $f$ is $\eta$-QM.

Proof. By 5.18, the implication $(2) \Rightarrow(1)$ is true in all Banach spaces. Assume that $f$ is $\varphi$-FQC. We may assume that $G \neq R^{1}, G^{\prime} \neq R^{1}$. By 4.9 , the Möbius map $u(x)=x /|x|^{2}$ is fully $4-\mathrm{QH}$ in $R^{1} \backslash\{0\}$. Using this and similarity maps we can normalize the situation to the case where $G=G^{\prime}=(0, \infty)$ and $f$ is increasing. It suffices to show that $f$ is weakly $H$-QS, which means the classical Beurling-Ahlfors condition.

Suppose that $x \in G$ and $t>0$ with $x-t \in G$. We apply solidity in the domains $D=(x-t, \infty)$ and $f D=(f(x-t), \infty)$. We have

$$
k_{D}(x, x+t)=\ln 2,
$$




$$
k_{f D}(f(x), f(x+t))=\ln \frac{f(x+t)-f(x-t)}{f(x)-f(x-t)}
$$

which imply

$$
\rho=\frac{f(x+t)-f(x)}{f(x)-f(x-t)} \leq e^{\varphi(\ln 2)} .
$$

To obtain a lower bound for $\rho$ we may assume $\rho \leq \frac{1}{2}$. Then 2.5 yields

$$
k_{f D}(f(x), f(x+t)) \leq 2 \rho,
$$

and hence $\rho \geq \varphi^{-1} \frac{1}{2} \ln 2$. $\square$

6.8. Remark. The one-dimensional case differs in many ways from the higherdimensional case. For example, if $f$ is a real $C^{1}$-function with positive derivative, then $H(x, f)=1$ for all $x$. Since $f$ need not be QM, Theorem 6.4 is not valid for $n=1$. We next give an example which shows that a fully $\varphi$-semisolid map need not be $\psi$-FQC with $\psi=\psi_{\varphi}$.

Let $0<\alpha<\frac{1}{2}$, let $G=(-1,1)$, and let $f: G \rightarrow G$ be the homeomorphism which sends $-\frac{1}{2}, 0, \frac{1}{2}$ to $-\alpha, 0, \alpha$ and is Möbius on the intervals $(-1,0)$ and $(0,1)$. Since $k(-\alpha, \alpha) \rightarrow 0$ as $\alpha \rightarrow 0, f$ is not $\varphi$-solid with a universal $\varphi$. However, with the aid of 4.6 one can show that $f$ is fully 4-Lipschitz in the QH metric.

The reason for the special properties of the case $\operatorname{dim} E=1$ seems to be the fact that balls have nonconnected boundaries and complements.

\section{Further results and open problems}

7.1. Announcements. In the second part [V $\left.\ddot{\mathrm{a}}_{4}\right]$ of this investigation we shall mainly consider the boundary behavior of FQC and solid maps. For example, an isolated boundary point is removable for these classes. We shall also develop the theory of uniform domains in Banach spaces. If $G$ and $G^{\prime}$ are $c$-uniform and $f: G \rightarrow G^{\prime}$ is $\varphi$-FQC, then $f$ is $\eta$-quasimöbius with $\eta=\eta(\varphi, c)$. In particular, $f$ extends to a homeomorphism $\bar{f}: \bar{G} \rightarrow \bar{G}^{\prime}$. If $f$ is only $\varphi$-solid, $f$ still has this homeomorphic extension, and the induced map $\partial G \rightarrow \partial G^{\prime}$ is $\eta$-quasimöbius. We also prove a reflection principle for FQC maps.

7.2. Open problems. We list some open questions.

1. Is a fully $\varphi$-semisolid map $\varphi_{1}$-FQC with $\varphi_{1}=\varphi_{1}(\varphi)$ ?

2. Suppose that $f: G \rightarrow G^{\prime}$ is a homeomorphism and that each point in $G$ has a neighborhood in which $f$ is $\varphi$-FQC. Is $f \varphi_{1}$-FQC with $\varphi_{1}=\varphi_{1}(\varphi)$ ?

3. Suppose that $H(x, f) \leq H_{0}$ for all $x \in G$. Is $f \varphi$-FQC with $\varphi=\varphi\left(H_{0}\right)$ ?

4. Is an FQC map somewhere differentiable? See 7.3.

5. Is an FQC map absolutely continuous on some line segment?

In the problems $1,2,3,5$ we assume that $\operatorname{dim} E \geq 2$, since in the onedimensional case the answer is known to be negative. The problems 1, 2, 3 are also open in the case $\operatorname{dim} E=n \geq 2$, since the known bounds depend on $n$. 
7.3. Addendum. An FQC map need not be differentiable anywhere. In fact, a bilipschitz map of the separable Hilbert space $E=l_{2}$ onto itself need not be differentiable anywhere. The following example was given to the author by D. Preiss: Define $g: E \rightarrow E$ by

$$
g x=\left(\left|x_{1}\right|,\left|x_{2}\right|, \ldots\right) .
$$

Then the map $f: E \rightarrow E$ defined by

$$
f x=x+g x / 2
$$

is 2-bilipschitz but nowhere differentiable.

On the other hand, Preiss $[\mathrm{Pr}]$ has recently proved that a Lipschitz map $f: l_{2} \rightarrow R^{1}$ is differentiable in a dense set.

\section{References}

[Ah] AHLfors, L.V.: Quasiconformal reflections. - Acta Math. 109, 1963, 291-301.

[AVV] Anderson, G.D., M.K. Vamanamurthy and M. Vuorinen: Dimension-free quasiconformal distortion in $n$-space. - Trans. Amer. Math. Soc. 297, 1986, 687-706.

[BA] Beurling, A., and L. AhLFors: The boundary correspondence under quasiconformal mappings. - Acta Math. 96, 1956, 125-142.

[Ca] Caraman, P.: Quasiconformal mappings in real normed spaces. - Rev. Roumaine Math. Pures Appl. 24, 1979, 33-78.

[Cr] CRistea, M.: Some conditions for quasiconformality. - Ann. Acad. Sci. Fenn. Ser. A I Math. 14, 1989, 345-350.

[Fe] Federer, H.: Geometric measure theory. - Springer-Verlag, Berlin, 1969.

[Ge] GeHRING, F.W.: The Carathéodory convergence theorem for quasiconformal mappings in space. - Ann. Acad. Sci. Fenn. Ser. A I Math. 336/11, 1963, 1-21.

[GO] Gehring, F.W., and B.G. OsGood: Uniform domains and the quasi-hyperbolic metric. - J. Analyse Math. 36, 1979, 50-74.

[GP] Gehring, F.W., and B.P. PaLka: Quasiconformally homogeneous domains. - J. Analyse Math. 30, 1976, 172-199.

[Gr] GRöTzsCH, H.: Über die Verzerrung bei schlichten nichtkonformen Abbildungen und über eine damit zusammenhängende Erweiterung des Picardschen Satzes. - Ber. Verh. Sächs. Akad. Wiss. Leipzig 80, 1928, 503-507.

[MS] Milman, V.D., and G. Schechtman: Asymptotic theory of finite dimensional normed spaces. - Lecture Notes in Mathematics 1200. Springer-Verlag, 1986.

[Po $\left.\mathrm{Po}_{1}\right]$ PoRru, G.: $\theta$-mappings and quasiconformal mappings in normed spaces. - Rend. Sem. Mat. Univ. Padova 57, 1977, 173-182.

$\left[\mathrm{Po}_{2}\right]$ PoRRU, G.: The boundary correspondence under mappings with bounded triangular dilatation in real normed spaces. - Analytic functions, Kozubnik 1979, Lecture Notes in Mathematics 798. Springer-Verlag, 1980, 392-401.

[Pr] Preiss, D.: Differentiability of Lipschitz functions on Banach spaces. - J. Funct. Anal. 91, $1990,312-345$.

[TV $\left.{ }_{1}\right]$ TUKIA, P., and J. VÄISÄLÄ: Quasisymmetric embeddings of metric spaces. - Ann. Acad. Sci. Fenn. Ser. A I Math. 5, 1980, 97-114. 
[TV $\left.{ }_{2}\right]$ TUKIA, P., and J. VÄISÄLÄ: Lipschitz and quasiconformal approximation and extension. - Ann. Acad. Sci. Fenn. Ser. A I Math. 6, 1981, 303-342.

$\left[\mathrm{TV}_{3}\right]$ TUKIA, P., and J. VÄISÄLÄ: Bilipschitz extensions of maps having quasiconformal extensions. - Math. Ann. 269, 1984, 561-572.

[Vä1] VÄISÄLÄ, J.: Quasimöbius maps. - J. Analyse Math. 44, 1984/85, 218-234.

[Vä] V̈̈̈s̈̈L̈̈, J.: Quasiconformal concordance. - Monatsh. Math. 107, 1989, 155-168.

[Vä3] VÄIs̈̈LÄ, J.: Quasiconformal maps of cylindrical domains. - Acta Math. 162, 1989, 201225.

[Vä4] V $\ddot{A} I S \ddot{A} L \ddot{A}$, J.: Free quasiconformality in Banach spaces II. - Preprint, University of Helsinki, 1990.

[Vu] Vuorinen, M.: Conformal geometry and quasiregular mappings. - Lecture Notes in Mathematics 1319. Springer-Verlag, Berlin-Heidelberg, 1988.

Helsingin yliopisto

Matematiikan laitos

Hallituskatu 15

SF-00100 Helsinki

Finland

Received 13 March 1990

Revised 1 October 1990 